\title{
A derivation of the mixed quantum-classical Liouville equation from the influence functional formalism
}

\author{
Qiang Shi and Eitan Geva \\ Department of Chemistry, University of Michigan, Ann Arbor, Michigan 48109-1055
}

(Received 17 March 2004; accepted 19 May 2004)

\begin{abstract}
We show that the mixed quantum-classical Liouville equation is equivalent to linearizing the forward-backward action in the influence functional. Derivations are provided in terms of either the diabatic or adiabatic basis sets. An application of the mixed quantum-classical Liouville equation for calculating the memory kernel of the generalized quantum master equation is also presented. The accuracy and computational feasibility of such an approach is demonstrated in the case of a two-level system nonlinearly coupled to an anharmonic bath. (C) 2004 American Institute of Physics. [DOI: 10.1063/1.1771641]
\end{abstract}

\section{INTRODUCTION}

Quantum effects play a central role in a variety of important processes that take place in condensed phase environments. ${ }^{1-3}$ Hence, the simulation of quantum dynamics in condensed phase hosts is one of the most important challenges facing theoretical chemistry. Whereas numerically exact classical molecular dynamics simulations are feasible for relatively complex many-body systems, the analogous numerically exact solution of the Schrödinger equation for such systems remains far beyond the reach of currently available computer resources, due to the exponential scaling of the computational effort with the number of degrees of freedom (DOF). A common approach for dealing with this difficulty is based on the observation that, in practice, one can often directly probe and/or manipulate only a few DOF. The subsystem subject to direct observation and/or manipulation may correspond to the reaction coordinate, a vibrational mode of a solute molecule, or the electronic DOF of an optically excited chromophore molecule in solution. Thus, it is worthwhile to consider a strategy that combines an accurate description of the subsystem, which will be referred to as the system from now on, with a minimal, yet accurate, treatment of the rest of the DOF, which will be referred to as the bath. The key to the success of such an approach relies on one's ability to accurately describe those aspects of the many-body bath dynamics which affect the system.

One way of approaching this challenge is based on the path-integral formulation of quantum mechanics, ${ }^{4-6}$ and introduces the influence of the bath in terms of an influence functional (IF). ${ }^{7}$ One of the most important advantages of this approach has to do with the fact that the exact IF can be obtained in closed form, in the case of linear coupling to a harmonic bath. ${ }^{8-11}$ This fact, in conjunction with important algorithmic advances, such as the development of iterative tensor quasiadiabatic propagators by Makri and co-workers, has opened the door to numerically exact calculations of the reduced dynamics of this type of systems (as long as one can evaluate the remaining path integral over the system DOF). ${ }^{12-27}$ However, there are many important systems, e.g., liquid solutions, where it is difficult, and perhaps even im- possible, to map the bath Hamiltonian onto a harmonic one. To the best of our knowledge, the only attempt so far for calculating the IF in the case of nonlinear coupling to an anharmonic bath has been carried out by Makri and coworkers, who proposed using the forward-backward semiclassical initial-value-representation approximation for this purpose. ${ }^{28-30}$

An alternative to the IF approach may be based on solving the Nakajima-Zwanzig generalized quantum master equation (GQME) ${ }^{31-45}$ which also provides an exact description of the system dynamics. In this equation, the influence of the bath on the system is given in terms of a memory kernel superoperator. The latter is analogous to the IF in the sense that it contains all the information needed in order to account for the influence of the bath on the system dynamics. In a previous paper, ${ }^{45}$ we presented a new framework for calculating the Nakajima-Zwanzig memory kernel, without resorting to the commonly made assumption of weak system-bath coupling. The strategy that we proposed is based on expressing this kernel in terms of two-time systemdependent bath correlation functions (SDBCFs). We have also proposed to approximate the SDBCFs by using the Meyer-Miller (MM) mapping for the system DOF, ${ }^{46-53}$ and linearizing the forward-backward (FB) action ${ }^{54-56}$ in the corresponding path-integral expression. ${ }^{57}$ The resulting linearized semiclassical Meyer-Miller (LSC-MM) approximation was found to perform rather well when applied to benchmark models. It should also be noted that a similar linearization approximation has been observed to lead to accurate results when used for calculating reaction rate constants, ${ }^{58}$ highfrequency vibrational energy relaxation rate constants, ${ }^{59,60}$ and nonradiative electronic relaxation rate constants. ${ }^{61,62}$

Another approach, which received much attention, is based on a mixed quantum-classical treatment. In this case, the system is treated quantum mechanically, while the bath is treated in a classical-like, trajectory-based, manner. ${ }^{63} \mathrm{~A}$ number of such mixed quantum-classical methodologies have been proposed, including ones based on a mean-field approach, ${ }^{64,65}$ surface-hopping, ${ }^{66-70}$ and hybrids that combine those two strategies. ${ }^{71-73}$ More recently, Martens and 
co-workers have proposed an approach based on a mixed quantum-classical Liouville (MQCL) equation. ${ }^{74-77}$ Over the last few years, this approach has been further explored and developed by several groups. ${ }^{78-83}$ Within this approach, a classical-like bath phase-space density $\sigma_{i j}(Q, P, t)$ is associated with each of the elements of the system reduced density matrix $\langle i|\hat{\sigma}| j\rangle$. The mixed quantum-classical equation of motion of $\sigma_{i j}(Q, P ; t)$ can be derived in several ways (e.g., by replacing the commutators by the corresponding Poisson brackets, performing a partial Wigner transform over the bath DOF, and taking the $\hbar \rightarrow 0$ limit with respect to them, etc.), and is referred to as the MQCL equation. Important recent advances by Martens et al. ${ }^{77,84}$ Santer, Manthe, and Stock, ${ }^{79}$ Nielsen, Kapral, and Ciccotti, ${ }^{81}$ and Wan and Schofield, ${ }^{82,83}$ have led to the development of practical stochastic trajectory algorithms for solving the MQCL equation. Although the computational cost involved in solving the MQCL equation is higher than that involved in either meanfield or surface-hopping techniques, it has been observed to be accurate in cases where those other techniques fail..$^{79,82,83}$

In this paper, we establish a general relationship between the IF formalism, the linearization approximation, and the MQCL equation. We will also propose a scheme for extending the applicability of the MQCL equation to longer times, with the help of the GQME formalism. Our main goals are as follows.

(i) To establish a relationship between the IF and MQCL approaches, by showing that linearizing the FB action in the exact path-integral expression for the IF is equivalent to the MQCL equation.

(ii) To develop an approach which restricts the use of the MQCL equation to calculating the short-lived memory kernel of the GQME. Subsequently, one can simulate relatively slow nonequilibrium relaxation processes by solving the GQME in a numerically exact manner.

The structure of the remainder of this paper is as follows. In Secs. II and III, we derive the MQCL equation, by linearizing the FB action in the IF in the case of diabatic and adiabatic basis sets, respectively. Section IV describes the calculation of the GQME memory kernel, which is based on solving the MQCL equation. The main results are summarized and discussed in Sec. V. A more detailed outline of the derivations is provided in two appendixes.

\section{THE DIABATIC CASE}

We consider a situation where a system of free Hamiltonian $\hat{H}_{s}$ is coupled to a bath of free Hamiltonian $\hat{H}_{b}$ with the coupling Hamiltonian $\hat{V}_{b s}$, such that the overall Hamiltonian is given by

$$
\hat{H}=\hat{H}_{s}+\hat{H}_{b}+\hat{V}_{b s} .
$$

We assume that the bath under consideration can be described in terms of $N_{b}$ nuclear DOF, such that

$$
\hat{H}_{b}=\sum_{j=1}^{N_{b}} \frac{\left[\hat{P}^{(j)}\right]^{2}}{2 M^{(j)}}+V_{b}(\hat{\mathbf{Q}}),
$$

where $\quad \hat{\mathbf{Q}}=\left(\hat{Q}^{(1)}, \ldots, \hat{Q}^{\left(N_{b}\right)}\right), \quad \hat{\mathbf{P}}=\left(\hat{P}^{(1)}, \ldots, \hat{P}^{\left(N_{b}\right)}\right), \quad$ and $\left(M^{(1)}, \ldots, M^{\left(N_{b}\right)}\right)$ are the corresponding coordinates, momenta, and masses, respectively. We assume that the dependence of $\hat{V}_{b s}$ on the bath DOF can be given in terms of $\hat{\mathbf{Q}}$. We also assume that the dependence of $\hat{V}_{b s}$ on the system DOF can be given in terms of a single system operator $\hat{S}$ such that $\hat{V}_{b s}=\hat{V}_{b s}(\hat{\mathbf{Q}}, \hat{S})$. Finally, let $\left\{\left|s_{j}\right\rangle \mid j=1, \ldots, n\right\}$ be the eigenbasis of the operator $\hat{S}$, such that $\hat{S}\left|s_{j}\right\rangle=s_{j}\left|s_{j}\right\rangle$, and therefore $\hat{V}_{b s}(\hat{\mathbf{Q}}, \hat{S})\left|s_{j}\right\rangle=\hat{V}_{b s}\left(\hat{\mathbf{Q}}, s_{j}\right)\left|s_{j}\right\rangle$.

The state of the overall system at time $t$ is given by the density operator

$$
\hat{\rho}(t)=e^{-i \hat{H} t / \hbar} \hat{\rho}(0) e^{i \hat{H} t / \hbar},
$$

where the initial state,

$$
\hat{\rho}(0)=\hat{\rho}_{b}(0) \otimes \hat{\sigma}(0),
$$

assumes a factorized form $\left[\hat{\rho}_{b}(0)\right.$ and $\hat{\sigma}(0)$ correspond to the density operators that represent the initial states of the bath and the system, respectively]. The sought after state of the system at time $t$ can then be described by the reduced density operator

$$
\hat{\sigma}(t)=\operatorname{Tr}_{b}[\hat{\rho}(t)]=\operatorname{Tr}_{b}\left[e^{-i \hat{H} t / \hbar} \hat{\rho}_{b}(0) \otimes \sigma(0) e^{i \hat{H} t / \hbar}\right],
$$

where $\operatorname{Tr}_{b}$ stands for a partial trace over the bath DOF.

For the sake of simplicity, the remainder of the derivation will be presented in terms of a 1D bath, such that $N_{b}$ $=1, \mathbf{Q} \rightarrow Q^{(1)} \rightarrow Q, \mathbf{P} \rightarrow P^{(1)} \rightarrow P, M^{(1)} \rightarrow M$ (the results can be generalized to the case of a multidimensional bath in a straightforward manner). As is well known, $\hat{\sigma}(t)$ can be represented by a matrix in terms of the basis set $\left\{\left|s_{j}\right\rangle \mid j\right.$ $=1, \ldots, n\}$. The elements of this matrix can be given in terms of the following path-integral expression. ${ }^{7,24,29,85}$

$$
\begin{aligned}
\left\langle s_{i}|\hat{\sigma}(t)| s_{j}\right\rangle= & \sum_{s_{0}^{+}} \cdots \sum_{s_{N-1}^{+}} \sum_{s_{N-1}^{-}} \cdots \sum_{s_{0}^{-}}\left\langle s_{N}^{+}\left|e^{-i \epsilon \hat{H}_{s} / \hbar}\right| s_{N-1}^{+}\right\rangle \cdots \\
& \times\left\langle s_{1}^{+}\left|e^{-i \epsilon \hat{H}_{s} / \hbar}\right| s_{0}^{+}\right\rangle\left\langle s_{0}^{+}|\hat{\sigma}(0)| s_{0}^{-}\right\rangle \\
& \times\left\langle s_{0}^{-}\left|e^{i \epsilon \hat{H}_{s} / \hbar}\right| s_{1}^{-}\right\rangle \cdots\left\langle s_{N-1}^{-}\left|e^{i \epsilon \hat{H}_{s} / \hbar}\right| s_{N}^{-}\right\rangle \\
& \times F\left[s_{0}^{ \pm}, \cdots, s_{N}^{ \pm}\right] .
\end{aligned}
$$

Here, $s_{i}=s_{N}^{+}, s_{j}=s_{N}^{-}, \epsilon=t / N$, and $F\left[s_{0}^{ \pm}, \ldots, s_{N}^{ \pm}\right]$is the IF, explicitly given by

$$
\begin{aligned}
F\left[s_{0}^{ \pm}, \ldots, s_{N}^{ \pm}\right]= & \left(\frac{M}{2 \pi \hbar \epsilon}\right)^{N} \int d Q_{0}^{+} \ldots \int d Q_{N-1}^{+} \int d Q_{N} \\
& \int d Q_{N-1}^{-} \ldots \int d Q_{0}^{-} \\
& \left\langle Q_{0}^{+}\left|\hat{\rho}_{b}(0)\right| Q_{0}^{-}\right\rangle e^{i\left(S_{N}^{+}-S_{N}^{-}\right) / \hbar},
\end{aligned}
$$


where

$$
S_{N}^{ \pm}=\epsilon \sum_{j=0}^{N-1} \frac{M}{2}\left(\frac{Q_{j+1}^{ \pm}-Q_{j}^{ \pm}}{\epsilon}\right)^{2}-V_{b}\left(Q_{j}^{ \pm}\right)-V_{b s}\left(Q_{j}^{ \pm}, s_{j}^{ \pm}\right),
$$

and $Q_{N}^{ \pm}=Q_{N}$.

It should be noted that Eq. (6) is based on the quasiadiabatic partitioning of the overall Hamiltonian into $\hat{H}_{s}$ (the free system Hamiltonian) and $\hat{H}_{\mathrm{env}}=\hat{H}_{b}+\hat{V}_{b s}$. The dependence on the bath DOF is restricted to the latter, and ends up being integrated over in the IF. As a result, one can use a bathindependent (diabatic) basis set in order to represent the system density matrix. The quasiadiabatic partitioning is expected to be particularly efficient (in the sense that relatively long time steps may be used) in cases where the bath dynamics is much faster than the system's dynamics. Under those circumstances, the bath DOF rapidly adjusts itself to the system displacement, such that the dependence of $\hat{H}_{\text {env }}$ on the system DOF is truly parametric.

In the next step, we apply the linearization approximation to the FB action in Eq. (7). More specifically, we assume that the dominant contributions to the IF arise from FB paths that are relatively close to each other, such that $S_{N}^{+}-S_{N}^{-}$can be replaced by its first-order expansion in terms of the deviation between the forward and backward paths. The actual derivation is similar to that involved in applying the same approximation to nonadiabatic correlation functions, and was described in detail elsewhere. ${ }^{57,62,86}$ Following the same procedure and making the transition from discrete time to continuous time leads to the following approximation for the IF:

$$
\begin{aligned}
F\left[s_{0}^{ \pm}, \ldots, s_{N}^{ \pm}\right] \approx & F_{L}\left[s_{0}^{ \pm}, \ldots, s_{N}^{ \pm}\right] \\
= & \frac{1}{2 \pi \hbar} \int d Q_{0} \int d P_{0} \rho_{b}^{W}\left(Q_{0}, P_{0} ; 0\right) \\
& \times \exp \left[-i \int_{0}^{t} d \tau U(\tau) / \hbar\right]
\end{aligned}
$$

where

$$
\begin{aligned}
\rho_{b}^{W}\left(Q_{0}, P_{0} ; 0\right)= & \frac{1}{2 \pi \hbar} \int_{-\infty}^{\infty} d \Delta e^{-i P \Delta / \hbar} \\
& \times\left\langle Q+\Delta / 2\left|\hat{\rho}_{b}(0)\right| Q-\Delta / 2\right\rangle
\end{aligned}
$$

is the Wigner transform of the initial bath density operator,

$$
U(\tau)=V_{b s}\left[Q_{\tau}, s_{\tau}^{+}\right]-V_{b s}\left[Q_{\tau}, s_{\tau}^{-}\right],
$$

and the dynamics of $Q_{\tau}$ is dictated by the following, explicitly time-dependent, averaged classical Hamiltonian:

$$
H^{\mathrm{av}}(t)=\frac{P}{2 M}+V_{b}(Q)+\frac{1}{2}\left\{V_{b s}\left[Q, s_{t}^{+}\right]+V_{b s}\left[Q, s_{t}^{-}\right]\right\} .
$$

Substituting the linearized IF in Eq. (9) back into Eq. (6) then yields the following approximate expression for a system density operator matrix element:

$$
\begin{aligned}
\left\langle s_{i}|\hat{\sigma}(t)| s_{j}\right\rangle= & \sum_{s_{0}^{+}} \cdots \sum_{s_{N-1}^{+}} \sum_{s_{N-1}^{-}} \cdots \sum_{s_{0}^{-}} \int d Q_{0} \int d P_{0} \\
& \times \rho_{b}^{W}\left(Q_{0}, P_{0} ; 0\right) \exp \left[-i \int_{0}^{t} d \tau U(\tau) / \hbar\right] \\
& \times\left\langle s_{N}^{+}\left|e^{-i \epsilon \hat{H}_{s} / \hbar}\right| s_{N-1}^{+}\right\rangle \cdots\left\langle s_{1}^{+}\left|e^{-i \epsilon \hat{H}_{s} / \hbar}\right| s_{0}^{+}\right\rangle \\
& \times\left\langle s_{0}^{+}|\hat{\sigma}(0)| s_{0}^{-}\right\rangle\left\langle s_{0}^{-}\left|e^{i \epsilon \hat{H}_{s} / \hbar}\right| s_{1}^{-}\right\rangle \cdots \\
& \times\left\langle s_{N-1}^{-}\left|e^{i \epsilon \hat{H}_{s} / \hbar}\right| s_{N}^{-}\right\rangle .
\end{aligned}
$$

Equation (13) can be used as such, as long as the numerical evaluation of the path integral over the system DOF is feasible (which will be the case if the system can be described in terms of a relatively small basis set). However, our goal here is to show that the approximation embodied by Eq. (13) is in fact equivalent to that underlying the diabatic version of the MQCL equation. To this end, we associate a bath phase-space density $\sigma_{i j}(Q, P ; t)$ with each system density matrix element $\left\langle s_{i}|\hat{\sigma}(t)| s_{j}\right\rangle$ such that

$$
\left\langle s_{i}|\hat{\sigma}(t)| s_{j}\right\rangle=\int d Q \int d P \sigma_{i j}(Q, P ; t) .
$$

It should be noted that the expectation value at time $t$ of any system operator $\hat{A}$ can be written in terms of a bath phasespace average of the following form:

$$
\langle\hat{A}\rangle(t)=\operatorname{Tr}_{s}(\hat{\sigma}(t) \hat{A})=\int d Q \int d P \sum_{i, j} \sigma_{i j}(Q, P ; t) A_{j i},
$$

where $A_{j i}=\left\langle s_{j}|\hat{A}| s_{i}\right\rangle$.

According to Eq. (13), $\sigma_{i j}(Q, P ; t)$ may be written in the following form:

$$
\begin{aligned}
\sigma_{i j}(Q, P ; t)= & \sum_{s_{0}^{+}} \cdots \sum_{s_{N-1}^{+}} \sum_{s_{N-1}^{-}} \cdots \sum_{s_{0}^{-}} \int d Q_{0} \int d P_{0} \rho_{b}^{W} \\
& \times\left(Q_{0}, P_{0} ; 0\right) \exp \left[-i \int_{0}^{t} d \tau U(\tau)\right] \\
& \times\left\langle s_{N}^{+}\left|e^{-i \epsilon \hat{H}_{s} / \hbar}\right| s_{N-1}^{+}\right\rangle \cdots\left\langle s_{1}^{+}\left|e^{-i \epsilon \hat{H}_{s} / \hbar}\right| s_{0}^{+}\right\rangle \\
& \times\left\langle s_{0}^{+}|\hat{\sigma}(0)| s_{0}^{-}\right\rangle\left\langle s_{0}^{-}\left|e^{i \epsilon \hat{H}_{s} / \hbar}\right| s_{1}^{-}\right\rangle \cdots \\
& \times\left\langle s_{N-1}^{-}\left|e^{i \epsilon \hat{H}_{s} / \hbar}\right| s_{N}^{-}\right\rangle \delta\left(Q-Q_{t}\right) \delta\left(P-P_{t}\right),
\end{aligned}
$$

where $Q_{t}$ and $P_{t}$ are propagated classically based on the particular realization of the averaged Hamiltonian in Eq. (12) [it should be noted that Eq. (16) involves integration over all such possible realizations].

We next seek an equation of motion for $\sigma_{i j}(Q, P ; t)$. To this end, we note that 


$$
\begin{aligned}
\sigma_{i j}(Q, P ; t+\epsilon)= & \sum_{k, l} \int d Q_{t} \int d P_{t} \sigma_{k l}\left(Q_{t}, P_{t} ; t\right) \\
& \times \exp \left[-i \int_{t}^{t+\epsilon} d \tau U(\tau) / \hbar\right] \\
& \times\left\langle s_{i}\left|e^{-i \epsilon \hat{H}_{s} / \hbar}\right| s_{k}\right\rangle\left\langle s_{l}\left|e^{i \epsilon \hat{H}_{s} / \hbar}\right| s_{j}\right\rangle \\
& \times \delta\left(Q-Q_{t+\epsilon}\right) \delta\left(P-P_{t+\epsilon}\right) .
\end{aligned}
$$

The equation of motion for $\sigma_{i j}(Q, P ; t)$ is then obtained from Eq. (17), with the help of the identity $d \sigma_{i j}(Q, P ; t) / d t$ $=\lim _{\epsilon \rightarrow 0}\left[\sigma_{i j}(Q, P ; t+\epsilon)-\sigma_{i j}(Q, P ; t)\right] / \epsilon$. An outline of the derivation is given in Appendix A, and only the final result is given below,

$$
\begin{aligned}
\frac{\partial \sigma_{i j}(Q, P ; t)}{\partial t}= & \left\{H_{i j}^{\mathrm{av}}, \sigma_{i j}(Q, P ; t)\right\}-\frac{i}{\hbar}\left[V_{b s}\left(Q, s_{i}\right)\right. \\
& \left.-V_{b s}\left(Q, s_{j}\right)\right] \sigma_{i j}(Q, P ; t) \\
& -\frac{i}{\hbar} \sum_{k}\left[\left\langle s_{i}\left|\hat{H}_{s}\right| s_{k}\right\rangle \sigma_{k j}(Q, P ; t)\right. \\
& \left.-\sigma_{i k}(Q, P ; t)\left\langle s_{k}\left|\hat{H}_{s}\right| s_{j}\right\rangle\right] .
\end{aligned}
$$

Here,

$$
\begin{aligned}
\left\{H_{i j}^{\mathrm{av}}, \sigma_{i j}(Q, P ; t)\right\}= & \frac{\partial H_{i j}^{\mathrm{av}}}{\partial Q} \frac{\partial \sigma_{i j}(Q, P ; t)}{\partial P} \\
& -\frac{\partial H_{i j}^{\mathrm{av}}}{\partial P} \frac{\partial \sigma_{i j}(Q, P ; t)}{\partial Q}
\end{aligned}
$$

is the classical Poisson bracket, and

$$
H_{i j}^{\mathrm{av}}=\frac{P^{2}}{2 M}+V_{i j}^{\mathrm{av}}(Q),
$$

where

$$
V_{i j}^{\mathrm{av}}(Q)=V_{b}(Q)+\frac{1}{2}\left[V_{b s}\left(Q, s_{i}\right)+V_{b s}\left(Q, s_{j}\right)\right] .
$$

Equation (18) corresponds to the MQCL equation, in terms of the diabatic basis set $\left\{\left|s_{j}\right\rangle \mid j=1, \ldots, n\right\}$. The emerging description associates a different potential energy surface $\left\{V_{i j}^{\text {av }}(Q)\right\}$ with each of the $n^{2}$ states in the system's Liouville space, $\left.{ }^{87}\{|i, j\rangle\rangle|\leftrightarrow| i\right\rangle\langle j||, i, j=1, \ldots, n\}$. The three terms on the RHS of Eq. (18) correspond to (in the order of appearance): (1) propagation of the bath phase-space density $\sigma_{i j}(Q, P ; t)$ on the potential energy surface $V_{i j}^{\mathrm{av}}(Q) ;(2)$ accumulation of phase during this single surface propagation (in the off-diagonal case, $i \neq j$ ); (3) transitions between surfaces, where the coupling arises from the off-diagonal matrix elements of the system Hamiltonian, $\left\{\left\langle s_{i}\left|\hat{H}_{s}\right| s_{j}\right\rangle, i \neq j\right\}$. It should also be noted that the factorized initial state in Eq. (4) implies that

$$
\left\langle s_{i}|\hat{\sigma}(0)| s_{j}\right\rangle=\int d Q \int d P \rho_{b}^{W}(Q, P ; 0)\left\langle s_{i}|\hat{\sigma}(0)| s_{j}\right\rangle,
$$

which implies that

$$
\sigma_{i j}(Q, P ; t=0)=\rho_{b}^{W}(Q, P ; 0)\left\langle s_{i}|\hat{\sigma}(0)| s_{j}\right\rangle .
$$

\section{THE ADIABATIC CASE}

The derivation of the diabatic MQCL equation in the preceding section was based on the quasiadiabatic partitioning of the overall Hamiltonian into $\hat{H}_{s}$ and $\hat{H}_{\mathrm{env}}=\hat{H}_{b}$ $+\hat{V}_{b s}$. In this section we consider an alternative partitioning of the overall Hamiltonian, which will lead to the adiabatic MQCL equation. To this end, let

$$
\hat{H}_{a}=\hat{H}_{s}+\hat{V}_{b s},
$$

such that $\hat{H}=\hat{H}_{a}+\hat{H}_{b}$. Also, define an adiabatic system basis set that consists of the eigenstates of $\hat{H}_{a}$, such that

$$
\hat{H}_{a}(Q)|k(Q)\rangle=E_{k}(Q)|k(Q)\rangle, \quad k=1, \ldots, n .
$$

It should be noted that the adiabatic basis functions and corresponding energy levels are parametrically dependent on the bath coordinate $Q$.

In the next step, we take the partial Wigner transform, over the bath DOF, of the overall density operator, ${ }^{78}$

$\hat{\sigma}^{W}(Q, P ; t)=\frac{1}{2 \pi \hbar} \int d \Delta e^{-i P \Delta / \hbar}\langle Q+\Delta / 2|\hat{\rho}(t)| Q-\Delta / 2\rangle$.

It should be noted that $\hat{\sigma}^{W}(Q, P, t)$ is a system operator, which can be represented by a matrix in terms of the basis set $\{|k(Q)\rangle\}$, such that the characteristic matrix element is given by

$$
\begin{aligned}
\sigma_{i j}^{W}(Q, P ; t)= & \left\langle i(Q)\left|\hat{\sigma}_{i j}^{W}(Q, P, t)\right| j(Q)\right\rangle \\
= & \frac{1}{2 \pi \hbar} \int d \Delta e^{-i P \Delta / \hbar}\langle i(Q), Q \\
& +\Delta / 2|\hat{\rho}(t)| j(Q), Q-\Delta / 2\rangle .
\end{aligned}
$$

It should be noted that the $Q$ dependence of $\sigma_{i j}^{W}(Q, P ; t)$ arises from two sources, namely, from that of $\hat{\sigma}^{W}(Q, P ; t)$ and from that of the basis set functions $|i(Q)\rangle$ and $|j(Q)\rangle$.

Importantly, knowledge of the matrix $\left\{\sigma_{i j}^{W}(Q, P ; t)\right\}$ is equivalent to that of the standard reduced density matrix $\hat{\sigma}$. More specifically, the expectation value of any system operator $\hat{A}$ at time $t$ can be obtained from $\left\{\sigma_{i j}^{W}(Q, P ; t)\right\}$ via the following identity:

$$
\begin{aligned}
\langle\hat{A}\rangle(t) & =\int d Q \int d P \operatorname{Tr}_{s}\left[\hat{\sigma}^{W}(Q, P ; t) \hat{A}\right] \\
& =\int d Q \int d P \sum_{i j} \sigma_{i j}^{W}(Q, P ; t) A_{j i}(Q),
\end{aligned}
$$

where $A_{j i}(Q)=\langle j(Q)|\hat{A}| i(Q)\rangle$.

Our goal in this section is to derive an equation of motion for $\sigma_{i j}^{W}(Q, P ; t)$, in a manner which is analogous to linearizing the FB action of the IF in the diabatic case. To this end, we note that $\sigma_{i j}^{W}(Q, P ; t+\epsilon)$ can be written in terms of $\sigma_{i j}^{W}(Q, P ; t)$, 


$$
\begin{aligned}
\sigma_{i j}^{W}(Q, P ; t+\epsilon)= & \frac{1}{2 \pi \hbar} \int d \Delta e^{-i P \Delta / \hbar}\left\langle i(Q), Q+\Delta / 2\left|e^{-i \epsilon \hat{H}_{b} / \hbar} e^{-i \epsilon \hat{H}_{a} / \hbar} \hat{\rho}(t) e^{i \epsilon \hat{H}_{a} / \hbar} e^{i \epsilon \hat{H}_{b} / \hbar}\right| j(Q), Q-\Delta / 2\right\rangle \\
= & \frac{1}{2 \pi \hbar} \sum_{k, l, m, n} \int d Q^{\prime} \int d P^{\prime} \int d \Delta \int d \Delta^{\prime} e^{-i P \Delta / \hbar} e^{i P^{\prime} \Delta^{\prime} / \hbar}\left\langle i(Q) \mid l\left(Q^{\prime}\right)\right\rangle \\
& \times\left\langle l\left(Q^{\prime}\right)\left|e^{-i \epsilon \hat{H}_{a}\left(Q^{\prime}+\Delta^{\prime} / 2\right) / \hbar}\right| k\left(Q^{\prime}\right)\right\rangle\left\langle Q+\Delta / 2\left|e^{-i \epsilon \hat{H}_{b} / \hbar}\right| Q^{\prime}+\Delta^{\prime} / 2\right\rangle \sigma_{k m}^{W}\left(Q^{\prime}, P^{\prime} ; t\right) \\
& \times\left\langle Q^{\prime}-\Delta^{\prime} / 2\left|e^{i \epsilon \hat{H}_{b} / \hbar}\right| Q-\Delta / 2\right\rangle\left\langle m\left(Q^{\prime}\right)\left|e^{i \epsilon \hat{H}_{a}\left(Q^{\prime}-\Delta^{\prime} / 2\right) / \hbar}\right| n\left(Q^{\prime}\right)\right\rangle\left\langle n\left(Q^{\prime}\right) \mid j(Q)\right\rangle,
\end{aligned}
$$

where we have used the inverse Wigner transform,

$$
\begin{gathered}
\left\langle k\left(q^{\prime}\right), Q^{\prime}+\Delta^{\prime} / 2|\hat{\rho}(t)| m\left(Q^{\prime}\right), Q^{\prime}-\Delta^{\prime} / 2\right\rangle \\
=\int d P^{\prime} e^{i P^{\prime} \Delta^{\prime} / \hbar} \sigma_{k m}^{W}\left(Q^{\prime}, P^{\prime} ; t\right) .
\end{gathered}
$$

The diagonal system overlap integrals that appear in Eq. (29) reduce to unity, in the limit $\epsilon \rightarrow 0$, due to the fact that $\langle n(Q) \mid \partial n(Q) / \partial Q\rangle \propto \partial\langle n(Q) \mid n(Q)\rangle / \partial Q=0$ (normalization),

$$
\begin{aligned}
\langle n(Q+\delta) \mid n(Q)\rangle & \approx\langle n(Q) \mid n(Q)\rangle+\langle\partial n(Q) / \partial Q \mid n(Q)\rangle \delta \\
& \rightarrow\langle n(Q) \mid n(Q)\rangle=1
\end{aligned}
$$

The off-diagonal system overlap integrals are evaluated as follows:

$$
\begin{aligned}
& \langle n(Q+\delta) \mid m(Q)\rangle \\
& \quad \approx\langle n(Q) \mid m(Q)\rangle+\langle\partial n(Q) / \partial Q \mid m(Q)\rangle \delta \\
& \quad=\langle\partial n(Q) / \partial Q \mid m(Q)\rangle \frac{P_{t}}{M} \epsilon
\end{aligned}
$$

$$
\begin{aligned}
& \langle n(Q) \mid m(Q+\delta)\rangle \\
& \quad \approx\langle n(Q) \mid m(Q)\rangle+\langle n(Q) \mid \partial m(Q) / \partial Q\rangle \delta \\
& \quad=\langle n(Q) \mid \partial m(Q) / \partial Q\rangle \frac{P_{t}}{M} \epsilon,
\end{aligned}
$$

where $P_{t}=M \delta / \epsilon$. The diagonal system propagators that appear in Eq. (29) can be approximated by

$$
\begin{aligned}
& \left\langle n(Q)\left|e^{\mp i \hat{H}_{a}(Q \pm \Delta / 2) / \hbar}\right| n(Q)\right\rangle \\
& \approx\left\langle n(Q)\left|1 \mp \frac{i}{\hbar} \epsilon \hat{H}_{a}(Q \pm \Delta / 2)\right| n(Q)\right\rangle \\
& \approx 1 \mp \frac{i}{\hbar} \epsilon E_{n}(Q)-\frac{i}{\hbar} \epsilon\left\langle n(Q)\left|\frac{\partial V_{b s}(\hat{Q})}{\partial Q}\right| n(Q)\right\rangle \frac{\Delta}{2} \\
& \approx \exp \left\{\mp \frac{i}{\hbar} \epsilon E_{n}(Q)-\frac{i}{\hbar} \epsilon\left\langle n(Q)\left|\frac{\partial V_{b s}(\hat{Q})}{\partial Q}\right| n(Q)\right\rangle \frac{\Delta}{2}\right\},
\end{aligned}
$$

where the second equality involves the linearization approximation. Similarly, the off-diagonal system propagators that appear in Eq. (29) is approximated by

$$
\begin{aligned}
& \left\langle n(Q)\left|e^{\mp i \hat{H}_{a}(Q \pm \Delta / 2) / \hbar}\right| m(Q)\right\rangle \\
& \approx-\frac{i}{\hbar} \epsilon\left\langle n(Q)\left|\frac{\partial V_{b s}(\hat{Q})}{\partial Q}\right| m(Q)\right\rangle \frac{\Delta}{2} \\
& \quad \approx \exp \left\{-\frac{i}{\hbar} \epsilon\left\langle n(Q)\left|\frac{\partial V_{b s}(\hat{Q})}{\partial Q}\right| m(Q)\right\rangle \frac{\Delta}{2}\right\}-1,
\end{aligned}
$$

where, once again, linearization has been employed. Finally, the FB action in the bath propagators that appear in Eq. (29) is also linearized, such that they are approximated by

$$
\begin{aligned}
&\left\langle Q+\Delta / 2\left|e^{-i \epsilon \hat{H}_{b} / \hbar}\right| Q^{\prime}+\Delta^{\prime} / 2\right\rangle\left\langle Q^{\prime}-\Delta^{\prime} / 2\left|e^{i \epsilon \hat{H}_{b} / \hbar}\right| Q-\Delta / 2\right\rangle \\
& \approx \frac{M}{2 \pi \hbar \epsilon} \exp \left\{\frac{i}{\hbar}\left[P_{t}\left(\Delta-\Delta^{\prime}\right)-\epsilon V_{b}^{\prime}\left(Q^{\prime}\right) \Delta^{\prime}\right]\right\}
\end{aligned}
$$

The equation of motion for $\sigma_{i j}^{W}(Q, P ; t)$ is obtained from Eq. (29), via the identity $d \sigma_{i j}^{W}(Q, P ; t) / d t$ $=\lim _{\epsilon \rightarrow 0}\left[\sigma_{i j}^{W}(Q, P ; t+\epsilon)-\sigma_{i j}^{W}(Q, P ; t)\right] / \epsilon$, and with the help of Eqs. (31)-(35). An outline of the derivation is given in Appendix B, and only the final result is given below, 


$$
\begin{aligned}
\frac{\partial}{\partial t} \sigma_{i j}^{W}(Q, P ; t)= & -\left\{\frac{P}{M} \frac{\partial}{\partial Q}+\frac{F_{i}(Q)+F_{j}(Q)}{2} \frac{\partial}{\partial P}\right\} \sigma_{i j}^{W}(Q, P ; t)-i \omega_{i j}(Q) \sigma_{i j}^{W}(Q, P ; t) \\
& +\sum_{k \neq i}\left\{-\frac{P}{M} d_{i k}(Q)+\frac{1}{2}\left\langle i(Q)\left|\frac{\partial V_{b s}(Q, \hat{S})}{\partial Q}\right| k(Q)\right\rangle \frac{\partial}{\partial P}\right\} \sigma_{k j}^{W}(Q, P ; t) \\
& +\sum_{k \neq j}\left\{\frac{P}{M} d_{k j}(Q)+\frac{1}{2}\left\langle k(Q)\left|\frac{\partial V_{b s}(Q, \hat{S})}{\partial Q}\right| j(Q)\right\rangle \frac{\partial}{\partial P}\right\} \sigma_{i k}^{W}(Q, P ; t) .
\end{aligned}
$$

Here, $\omega_{i j}(Q)=\left[E_{i}(Q)-E_{j}(Q)\right] / \hbar$,

$$
F_{j}(Q)=-V_{b}^{\prime}(Q)-\left\langle j(Q)\left|\frac{\partial V_{b s}}{\partial Q}\right| j(Q)\right\rangle
$$

are the Hellmann-Feynman forces, and

$$
d_{m n}(Q)=\langle m(Q) \mid \partial n(Q) / \partial Q\rangle
$$

are the nonadiabatic coupling coefficients.

Equation (36) corresponds to the MQCL equation in the case of an adiabatic basis set. ${ }^{76,79-83}$ The emerging description is similar to that obtained in the case of the diabatic basis set, in that it associates a different potential energy surface,

$$
\begin{aligned}
V_{i j}(Q)= & V_{b}(Q)+\frac{1}{2}\left[\left\langle i(Q)\left|V_{b s}(Q, \hat{S})\right| i(Q)\right\rangle\right. \\
& \left.+\left\langle j(Q)\left|V_{b s}(Q, \hat{S})\right| j(Q)\right\rangle\right],
\end{aligned}
$$

with each of the $n^{2}$ states in the system's Liouville space, $\{|i(Q)\rangle\langle j(Q)|\leftrightarrow| i j\rangle\rangle \mid i, j=1, \ldots, n\}$. The three terms on the RHS of Eq. (18) correspond to (in the order of appearance): (1) classical propagation of the bath phase-space density $\sigma_{i j}^{W}(Q, P ; t)$ on the $V_{i j}(Q)$ surface; (2) accumulation of phase during this single surface propagation (in the case where $i \neq j$ ); (3) transitions between surfaces, where the nonadiabatic coupling arises from the off-diagonal matrix elements $\left\{\left\langle i(Q)\left|\partial V_{b s}(Q, \hat{S}) / \partial Q\right| j(Q)\right\rangle\right.$ and $\left\{d_{i j}\right\}$. It should also be noted that the factorized initial state in Eq. (4) implies that

$$
\sigma_{i j}^{W}(Q, P ; t=0)=\rho_{b}^{W}(Q, P ; 0)\langle i(Q)|\hat{\sigma}(0)| j(Q)\rangle .
$$

Equation (36) was derived based on the partitioning of the overall Hamiltonian into $\hat{H}_{a}=\hat{H}_{s}+\hat{V}_{b s}$ and $\hat{H}_{b}$. This partitioning dictated the use of an adiabatic system basis set, which is parametrically dependent on the bath coordinate. The main advantage of this approach has to do with the fact that it restricts the transitions between the surfaces to the, presumably localized, regions in space where there is significant overlap between $|i(Q)\rangle$ and $|j(Q)\rangle$. This results in the minimization of the branching of the trajectory taken by $Q$ between the various surfaces, which is desirable from the point of view of computational feasibility (see Sec. IV C). At the same time, the main disadvantage of the adiabatic MQCL approach has to do with the need to repeatedly diagonalize $\hat{H}_{a}$, on the fly, in order to adjust the basis set to variations in $Q$. While such a diagonalization can be performed analytically in the case of two- and three-level systems, it can become the computational bottleneck in cases involving many more states. The adiabatic representation is particularly suitable to cases where the system dynamics is much faster than the bath dynamics. Under those circumstances, the system DOF rapidly adjust themselves to the displacements of the bath, such that the dependence of $\hat{H}_{a}$ on the bath DOF is truly parametric. A prominent example correspond to the case where the system and bath correspond to electronic and nuclear DOFs, respectively.

\section{CALCULATION OF THE MEMORY KERNEL OF THE GENERALIZED QUANTUM MASTER EQUATION}

\section{A. The generalized quantum master equation}

A major weakness of almost all available approximate methods for simulating many-body quantum dynamics has to do with the fact that their accuracy and/or computational feasibility deteriorate with time. The derivation of the MQCL equation in Sec. II implies that it is equivalent to linearizing the IF. It should be noted that the accuracy of the linearization approximation is known to detcriorate with time..$^{57,59}$ Nevertheless, it is also known that the IF is characterized by a relatively short memory time in condensed phase systems. Thus, restricting the use of the linearization approximation to calculating the short-lived IF is more likely to yield an accurate description of the system relaxation at long times (in comparison to the direct application of this approximation). Unfortunately, the computational cost of solving the MQCL equation via the stochastic trajectory algorithm (see below) grows exponentially with time, which is attributed to inefficient sampling due to branching of the trajectories between the different surfaces. ${ }^{79}$ Thus, one expects that a direct application of the MQCL equation for simulating nonequilibrium dynamics will only be feasible in the case of relatively rapid relaxation processes. In this section, we propose a general methodology which is based on restricting the use of the MQCL equation to the calculation of the relatively shortlived memory kernel of the GQME. Simulation of nonequilibrium relaxation processes, which may be characterized by longer time scales, is then made possible by solving the GQME in a numerically exact manner.

The general theoretical framework for calculating the memory kernel has been described in previous papers, ${ }^{45,57}$ and will only be outlined below. The general approach is based on describing the system's dynamics in terms of the Nakajima-Zwanzig GQME,

$$
\frac{d}{d t} \hat{\sigma}(t)=-\frac{i}{\hbar}\left[\hat{H}_{s}, \hat{\sigma}(t)\right]-\int_{0}^{t} d \tau \mathcal{K}(\tau) \hat{\sigma}(t-\tau) .
$$


Here, $\mathcal{K}(\tau)$ is the memory kernel, whose explicit form is well known (e.g., see Ref. 45). $\mathcal{K}(\tau)$ is a system superoperator that can be represented by an $n^{2} \times n^{2}$ matrix in Liouville space. The input required for calculating the $n^{2} \times n^{2}$ matrix that represents $\mathcal{K}(\tau)$, in terms of the diabatic basis set of the system, $\left\{\left|s_{j}\right\rangle \mid j=1, \ldots, n\right\}$, is given by $\sim n^{4}$ SDBCFs of the following form: ${ }^{45}$

$$
\begin{aligned}
\langle\hat{\Gamma}(2, b, a, 1 ; \tau) \hat{\Lambda}\rangle_{\mathrm{eq}}= & \operatorname{Tr}\left\{\hat{\Lambda} \hat{\rho}_{b}^{\mathrm{eq}}\left|s_{1}\right\rangle\left\langle s_{2}\left|e^{i \hat{H} \tau / \hbar}\right| s_{b}\right\rangle\right. \\
& \left.\times\left\langle s_{a}\right| \hat{\Gamma} e^{-i \hat{H} \tau / \hbar}\right\} .
\end{aligned}
$$

Here, $\hat{\rho}_{b}^{\mathrm{eq}}=e^{-\beta \hat{H}_{b}} / \operatorname{Tr}_{b}\left[e^{-\beta \hat{H}_{b}}\right]$ is the equilibrium density operator of the free bath, and $\hat{\Lambda}, \hat{\Gamma}$ correspond to various bath operators which are given explicitly in Refs. 45 and 57. Importantly, correlation functions of the form of Eq. (42) are generally short lived, as is the memory kernel that can be obtained from them. Thus, approximating them via the MQCL equation, followed by a calculation of the memory kernel, via the procedure described in Refs. 45 and 57, and a numerically exact solution of the GQME, can make it possible to accurately simulate relatively slow nonequilibrium relaxation processes, which could not be described by direct application of the MQCL equation.

\section{B. The model}

We will now demonstrate this approach in the case of a two-level system (TLS) nonlinearly coupled to an anharmonic bath. The model employed is adopted from Ref. 57, where it was used for testing the LSC-MM approximation. It consists of a linear chain of 11 Helium atoms that lie along the $x$ axis, and an atom $A$, which is attached to one end of the chain. Atom $A$ and the 11th Helium atom at the other end of the chain are held fixed at $x=0$ and $x=\sigma_{\mathrm{He}-A}+10 \sigma_{\mathrm{He}-\mathrm{He}}$, respectively. The instantaneous positions of the first ten Helium atoms $(i=1,2, \ldots, 10)$ are given by $\left\{x_{i}=\sigma_{\mathrm{He}-A}+(i\right.$ $\left.-1) \sigma_{\mathrm{He}-\mathrm{He}}+\delta_{i}\right\}$. Only nearest neighbor interactions along the chain are taken into account. The TLS corresponds to an internal DOF of atom $A$, and the interaction between atom $A$ and the Helium atom attached to it depends on the internal state of atom $A$.

The bath Hamiltonian is given by

$$
\hat{H}_{b}=\sum_{k=1}^{10} \frac{\left(p^{(k)}\right)^{2}}{2 M_{\mathrm{He}}}+\frac{1}{2}\left[V_{+}(\hat{\mathbf{x}})+V_{-}(\hat{\mathbf{x}})\right]
$$

where,

$$
\begin{aligned}
V_{ \pm}= & v_{\mathrm{LJ}}^{\mathrm{He}-A}\left(\sigma_{\mathrm{He}-A}+\delta_{1} \pm r_{0}\right) \\
& +\sum_{i=1}^{10} v_{\mathrm{LJ}}^{\mathrm{He}-\mathrm{He}}\left(\sigma_{\mathrm{He}-\mathrm{He}}+\delta_{i+1}-\delta_{i}\right) .
\end{aligned}
$$

Here, $V_{ \pm}$correspond to the potential energy that correspond to the two states $| \pm\rangle$ of the TLS, and

$$
v_{\mathrm{LJ}}(r)=4 \epsilon\left[\left(\frac{\sigma}{r}\right)^{12}-\left(\frac{\sigma}{r}\right)^{6}\right]
$$

is the familiar Lennard-Jones (LJ) potential. $\sigma$ and $\epsilon$ are given by $\left\{\sigma_{\mathrm{He}-A}, \epsilon_{\mathrm{He}-A}\right\}$ and $\left\{\sigma_{\mathrm{He}-\mathrm{He}}, \epsilon_{\mathrm{He}-\mathrm{He}}\right\}$ for the $\mathrm{He}-A$, and $\mathrm{He}-\mathrm{He}$ interactions, respectively. The system Hamiltonian is given by

$$
\hat{H}_{s}=\Omega \hat{\sigma}_{x}+\left[\Delta+\frac{1}{2}\left\langle V_{+}(\hat{\mathbf{x}})-V_{-}(\hat{\mathbf{x}})\right\rangle_{\mathrm{eq}}\right] \hat{\sigma}_{z},
$$

where $\hat{\sigma}_{x}, \hat{\sigma}_{y}, \hat{\sigma}_{z}$ are the familiar Pauli operators, and $\langle\hat{\Gamma}\rangle_{\mathrm{eq}}=\operatorname{Tr}_{b}\left(\tilde{\rho}_{b}^{\mathrm{eq}} \hat{\Gamma}\right)$. The system-bath coupling is given by

$$
\hat{V}_{b s}=\hat{\Lambda}(\hat{\mathbf{x}}) \otimes \hat{\sigma}_{z},
$$

where

$$
\hat{\Lambda}=\frac{1}{2}\left[V_{+}(\hat{\mathbf{x}})-V_{-}(\hat{\mathbf{x}})-\left\langle V_{+}(\hat{\mathbf{x}})-V_{-}(\hat{\mathbf{x}})\right\rangle_{\mathrm{eq}}^{0}\right] .
$$

The diabatic basis is in this case given by the eigenfunctions of $\hat{\sigma}_{z}: \hat{\sigma}_{z}| \pm\rangle= \pm| \pm\rangle$.

Calculations pertaining to this model have been performed using the following values of the parameters: $T$ $=1 / k_{B} \beta=40 \mathrm{~K}, \quad \sigma_{\mathrm{He}-A}=4.944$ a.u., $\quad \sigma_{\mathrm{He}-\mathrm{He}}=4.310$ a.u., $\epsilon_{\mathrm{He}-\mathrm{A}} / k_{B}=25.1 \mathrm{~K}, \quad \epsilon_{\mathrm{He}-\mathrm{He}} / k_{B}=10.2 \mathrm{~K}, \Omega=1.0 \times 10^{-4}$ a.u., $\Delta=1.2 \times 10^{-4}$ a.u., and $r_{0}=0.2$ a.u. The local harmonic approximation, which is described in detail in Ref. 59, has been used in order to calculate the Wigner transform of operators of the form $\hat{\Lambda} \hat{\rho}_{b}^{\mathrm{eq}}$ [see Eq. (42)].

\section{Computer algorithm}

The theoretical framework for calculating the memory kernel was given in terms of a diabatic representation. ${ }^{45}$ It is therefore natural to use the diabatic MQCL equation for calculating it (although it should be noted that a calculation based on an adiabatic basis set would probably be more efficient). In this section, we outline the stochastic trajectory algorithm employed for solving the adiabatic MQCL equations. To this end, it is convenient to consider an $n^{2}$-dimensional vector $\vec{\sigma}(Q, P ; t)$ whose components are given by $\left\{\sigma_{i j}(Q, P ; t) \mid i, j=1, \ldots, n\right\}$. Equation (18) may then be put in the following form:

$$
\begin{aligned}
\frac{\partial}{\partial t} \vec{\sigma}(Q, P ; t) & =\left(\mathcal{L}_{\text {av }}+\mathcal{L}_{\text {phase }}+\mathcal{L}_{s}\right) \vec{\sigma}(Q, P ; t) \\
& =\mathcal{L} \vec{\sigma}(Q, P ; t),
\end{aligned}
$$

where $\mathcal{L}_{\text {av }}, \mathcal{L}_{\text {phase }}$, and $\mathcal{L}_{s}$ represent $n^{2} \times n^{2}$ matrices that correspond to the three terms on the RHS of Eq. (18), in the order in which they appear there. The propagation of $\vec{\sigma}(Q, P ; t)$ from time $t$ to time $t+\epsilon$, where $\epsilon$ is a small time step, can then be put in the following form:

$$
\vec{\sigma}(Q, P ; t+\epsilon)=e^{\epsilon \mathcal{L}_{s}} e^{\epsilon \mathcal{L}_{d}} \vec{\sigma}(Q, P ; t),
$$

where $\mathcal{L}_{d}=\mathcal{L}_{\text {av }}+\mathcal{L}_{\text {phase }}$.

Operating with $e^{\epsilon \mathcal{L}_{d}}$ on $\vec{\sigma}(Q, P ; t)$ is relatively straightforward because $\mathcal{L}_{d}$ corresponds to a diagonal $n^{2} \times n^{2}$ matrix. Thus, each of the bath phase-space densities $\left\{\sigma_{i j}(Q, P ; t)\right\}$ is independently propagated subject to the classical Hamiltonian $H_{i j}^{\text {av }}$, with the off-diagonal ones ( $i$ $\neq j$ ) also accumulating phase in the process. It should be noted that the bath is multidimensional in most cases of practical interest, which implies that propagating the actual phase-space densities $\left\{\sigma_{i j}(Q, P ; t)\right\}$ is not feasible. However, due to the classical nature of the dynamics, one may instead 
propagate $Q$ and $P$, and take advantage of the fact that $e^{\epsilon \mathcal{L}_{\mathrm{av}}} \sigma_{i j}[Q, P]=\sigma_{i j}[Q(t+\epsilon), P(t+\epsilon)]$, where $Q(t+\epsilon)$ and $P(t+\epsilon)$ are obtained by classical propagation, subject to the Hamiltonian $H_{i j}^{\mathrm{av}}$, and starting with $Q(t)=Q$ and $P(t)=P$.

Operating with $e^{\epsilon \mathcal{L}_{s}}$ involves mixing between the various bath phase-space densities $\left\{\sigma_{i j}(Q, P ; t)\right\}$. A stochastic branching algorithm similar to that used by Santer, Manthe, and Stock $^{79}$ can be used in order to accomplish this in terms of classical trajectories, rather than in terms of the actual phase-space densities. To this end, one defines the quantum amplitude for a transition, within a time step $\epsilon$, from one Liouville space state $\left.\left|s_{i}, s_{j}\right\rangle\right\rangle \leftrightarrow\left|s_{i}\right\rangle\left\langle s_{j}\right|$ to another Liouville space state $\left.\left|s_{k}, s_{l}\right\rangle\right\rangle \leftrightarrow\left|s_{k}\right\rangle\left\langle s_{l}\right|$,

$$
T_{k l \leftarrow i j}=\left\langle\left\langle s_{k}, s_{l}\left|e^{\epsilon \mathcal{L}_{s}}\right| s_{i}, s_{j}\right\rangle\right\rangle=\left\langle i\left|e^{-i \epsilon \hat{H}_{s} / \hbar}\right| k\right\rangle\left\langle l\left|e^{i \epsilon \hat{H}_{s}}\right| j\right\rangle .
$$

One then defines a transition probability as follows:

$$
p(k l \leftarrow i j)=\frac{|T(k l \leftarrow i j)|}{\sum_{m, n}|T(m n \longleftarrow i j)|} .
$$

Thus, operating with $e^{\epsilon \mathcal{L}_{s}}$ on $\vec{\sigma}(Q, P ; t)$ translates into random hops of the trajectory from the potential surface $V_{i j}^{\mathrm{av}}(Q)$, to another potential surface, $V_{k l}^{\mathrm{av}}(Q)$, with the probability for hopping within a time step given by $p_{k l \leftarrow i j}$ (the trajectory may also stay on the same potential surface if $\mathrm{kl}$ $=i j$ ). It is important to note that each trajectory accumulates a complex weighting factor, which is equal to one at $t=0$, and is multiplied by $T(k l \leftarrow i j) / p(k l \leftarrow i j)$ at each time step, based on the particular choice of a random hopping event.

The above discussion gives rise to the following numerical algorithm for solving the MQCL equation.

(1) For a given choice of a Liouville space state $\left.\left|s_{i}, s_{j}\right\rangle\right\rangle$, sample the initial values of the bath coordinate and momentum, $Q(0)$ and $P(0)$, respectively, from the Wigner distribution $\left[\hat{\rho}_{b}^{\mathrm{eq}}\right]^{W}(Q, P)$. It should be noted that, in the case of an anharmonic system, the Wigner transform can be calculated using the local harmonic approximation. ${ }^{59}$

(2) Propagate $Q(0)$ and $P(0)$ forward by one time step, on the potential surface $V_{i j}^{\mathrm{av}}(Q)$, to obtain $Q(\epsilon)$ and $P(\epsilon)$. Also, calculate the corresponding phase factor if $i \neq j$.

(3) Perform a random transition from the $V_{i j}^{\text {av }}(Q)$ surface to another $V_{k l}^{\mathrm{av}}(Q)$ surface, based on the probability $p(k l \leftarrow i j)$, and multiply the weight factor of the trajectory by $T(k l \leftarrow i j) / p(k l \leftarrow i j)$.

(4) Repeat the procedure in steps 2 and 3 at subsequent time steps.

(5) Sample over other values of $Q(0)$ and $P(0)$ and for other choices of initial Liouville space states.

(6) Compute the quantity of interest at each time step, by averaging over all the trajectories (also taking into account their accumulated phases and weight factors).

The algorithm described above is not feasible for long times, due to the multiplicity of hopping events, which leads to an exponential decrease of the weight factors of individual trajectories, and therefore to an exponential increase in the number of trajectories that one needs to sample in order to obtain a converged result. For the model under consideration, the overall time was set to $t=1.2 \times 10^{4}$ a.u., as dictated by the lifetime of the memory kernel. This time was split into 20

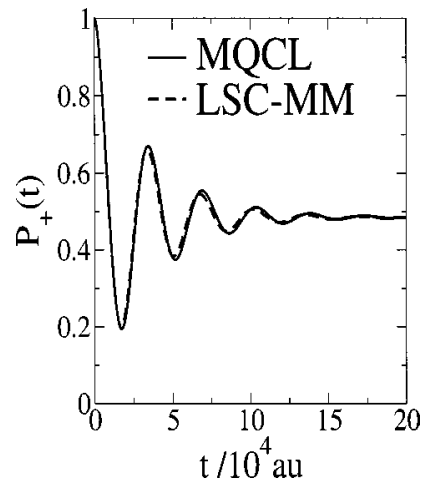

FIG. 1. The relaxation of $P_{+}(t)=\langle+|\hat{\sigma}(t)|+\rangle$ to equilibrium in the case of a TLS nonlinearly coupled to an anharmonic bath which consists of a chain of 11 Helium atoms. Shown are the results based on using either the MQCL equation (solid line), or LSC-MM method (dashed line) for calculating the memory kernel of the GQME.

time steps, and second order Trotter splitting was employed for the single time step propagator. $2 \times 10^{6}$ trajectories were found to be sufficient for obtaining a converged result for the required correlations functions, and the memory kernel that results from them. The procedure for obtaining the memory kernel from the SDBCFs, and subsequently simulating the system dynamics, is similar to that described in Ref. 45 .

\section{Results}

In Fig. 1, we show the relaxation of the excited state population, $P_{+}(t)=\langle+|\hat{\sigma}(t)|+\rangle$, to equilibrium, starting from the initial state, $\hat{\sigma}(0)=|+\rangle\langle+|$. The results obtained by solving the MQCL equation (solid line) are compared to those previously obtained, for the same model, by the LSC-MM method (dashed line). ${ }^{57}$ It is important to note that simulating this relaxation dynamics via a direct solution of the MQCL equation is prohibitively expensive, and was therefore not carried out in practice. It should also be noted that the MQCL and LSC-MM methods are rather different, despite the fact that they are both based on the linearization of the FB path-integral action. More specifically: (i) the MQCL equation is based on linearizing the IF, whereas the LSC-MM method is based on linearizing the SDBCFs; (ii) the MQCL approach treats the electronic DOF in terms of the original basis set, whereas the LSC-MM method is based on mapping it onto classical-like continuous DOFs. Despite of those differences, the MQCL and LSC-MM methods lead to results which are in good agreement with each other. It should be noted that although solving the MQCL equation is computationally more demanding, it has several advantages over the LSC-MM approach, namely, (a) the MQCL equation is exact in the case where the bath is harmonic (as long as the normal mode coordinates and frequencies in the ground and excited electronic states are the same); (b) in the case of anharmonic systems, the LSC-MM method has been observed to yield unstable trajectories, which had to be discarded in Ref. 57, while the MQCL method does not.

In the case of a TLS, it can be shown that out of the 16 matrix elements of the memory kernel, only four are independent. Those four independent elements can be chosen as: $\langle\langle+-|\mathcal{K}(\tau)|++\rangle\rangle,\langle\langle+-|\mathcal{K}(\tau)|--\rangle\rangle,\langle\langle+-|\mathcal{K}(\tau)|+-\rangle\rangle$, and 


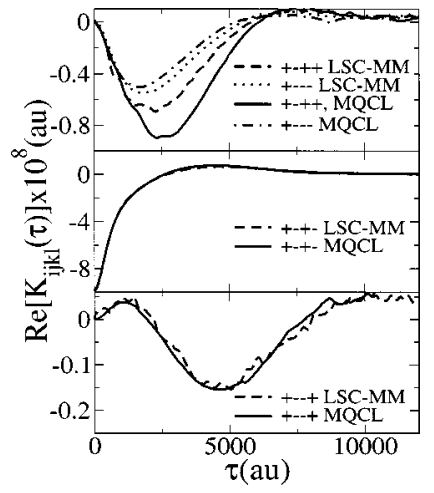

FIG. 2. The real parts of $\langle\langle+-|\mathcal{K}(\tau)|++\rangle\rangle$ and $\langle\langle+-|\mathcal{K}(\tau)|--\rangle\rangle$ (upper panel), $\langle\langle+-|\mathcal{K}(\tau)|+-\rangle\rangle$ (middle panel), and $\langle\langle+-|\mathcal{K}(\tau)|-+\rangle\rangle$ (lower panel), for a TLS coupled to a linear chain of 11 Helium atoms. Shown are the results obtained via the MQCL approximation, and LSC-MM method (the latter are adopted from Ref. 57).

$\langle\langle+-|\mathcal{K}(\tau)|-+\rangle\rangle{ }^{45}$ Their real and imaginary parts, as obtained via the MQCL and LSC-MM treatments, are shown in Figs. 2 and 3, respectively. It should be noted that the time scale of the memory kernel elements, which dictates the memory time of the bath, is an order of magnitude shorter than that of the system relaxation (see Fig. 1). This is the reason for why using the MQCL equation for calculating the memory kernel is feasible, whereas using it for directly simulating the system relaxation is prohibitively expensive. The agreement between the MQCL and LSC-MM results is clearly not as good as in the case of the actual system dynamics (see Fig. 1). This suggests that the actual system dynamics is not very sensitive to the fine details of the SDBCFs. This observation is also consistent with similar observations discussed in Ref. 57.

\section{SUMMARY}

Many advances in path-integral and mixed quantumclassical techniques for simulating many-body quantum dynamics have been made over the last few years. For the most part, those advances were made independently, and relatively little effort was put into understanding the relationship be-

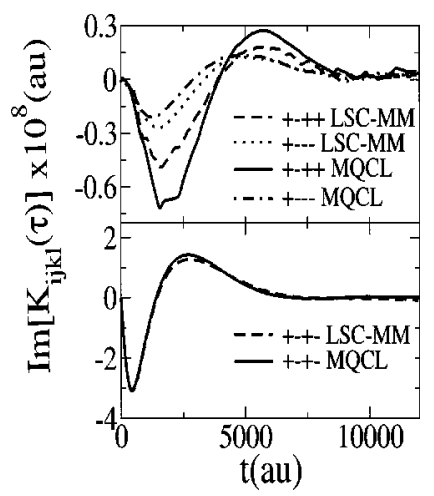

FIG. 3. The imaginary parts of $\langle\langle+-|\mathcal{K}(\tau)|++\rangle\rangle$ and $\langle\langle+-|\mathcal{K}(\tau)|--\rangle\rangle$ (upper panel), and $\langle\langle+-|\mathcal{K}(\tau)|+-\rangle\rangle$ (lower panel), for a TLS coupled to a linear chain of 11 Helium atoms. Note that $\operatorname{Im}\langle\langle+-|\mathcal{K}(\tau)|-+\rangle\rangle=0$ in this case (see lower panel of Fig. 2 for its real part). Shown are the results obtained via the MQCL approximation, and LSC-MM method (the latter are adopted from Ref. 57). tween those two approaches. The fundamental significance of establishing such relations is obvious. However, such relations are also desirable from a more practical point of view, as they shed light on different aspects of the underlying approximations, as well as promote the transfer of techniques between different approaches. In this paper, we established a general relationship between the path-integral IF and mixed quantum-classical Liouville descriptions of a system coupled to a bath. We have found that the dynamics dictated by the MQCL equation is equivalent to that dictated by a linearized IF. One implication is that well established concepts and techniques that were developed within the framework of the path-integral formalism can now be applied within the framework of a mixed quantum-classical treatment, and vice versa. For example, an interesting alternative to actually solving the MQCL equation, could be based on using pathintegral techniques in order to calculate the IF in Eq. (9). As for the MQCL equation, the computational cost involved in evaluating the path integral in Eq. (13) will grow exponentially with time. However, one could effectively restrict the calculation to a relatively short time, by taking advantage of the finite memory time of the bath. To this end, one could employ a strategy based on the iterative tensor propagator concept, similar to that employed by Makri and co-workers in the context of the semiclassical approximation for the $\mathrm{IF}^{28-30}$ It should be noted that such an approach would correspond to the path-integral analog of the GQME-based approach described above, which also takes advantage of the relatively short memory time of the bath. (A general discussion of the pros and cons of the GQME and IF approaches can be found in Ref. 45.) One could also go in the opposite direction, and explore other types of mixed quantumclassical equations of motion that may originate from other approximations for the IF.

We have also proposed a methodology which combines the MQCL equation with the GQME equation. This strategy circumvents the prohibitively high computational cost involved in solving the MQCL equation for long times, by only using it for calculating the, typically short-lived, memory kernel of the GQME. The memory kernel can then be used as input for a numerically exact solution of the GQME, which is feasible as long as the system basis set is not extensive. We have also reported a comparison between the MQCL and LSC-MM approximations as ways for calculating the memory kernel. In a previous study, we have found that several trajectories become unstable within the framework of the LSC-MM approximation, and needed to be discarded. This unphysical instability can be traced back to the MM mapping, and the number of trajectories exhibiting it was found to rapidly increase with time. ${ }^{57}$ The MQCL treatment describes the system in terms of the original basis set, and therefore does not suffer from this problem. At the same time, the MQCL equation is more demanding computationally, and especially so at long times. Both methods yielded almost identical results in the case of the model system studied in this paper. More applications would be needed in order to gain further insight into the optimal balance between accuracy, feasibility, and self-consistency of those two methods. It is nevertheless clear that pursuing such a MQCL- 
GQME approach would allow for the simulation of nonequilibrium quantum dynamics of a system with an arbitrary coupling to an anharmonic environment, beyond the domains of linear response theory and the Bloch-Redfield equation. $^{45,57}$ Many exciting applications fall into this category, including nonadiabatic dynamics and optical chromophore spectroscopy in liquid solution and other anharmonic media. Those and other applications are the subject of ongoing work in our group, and will be reported in future publications.

\section{ACKNOWLEDGMENT}

This project was supported by the National Science Foundation FOCUS Center, through Grant No. PHY0114336.

\section{APPENDIX A: THE DERIVATION OF THE MQCL EQUATION IN THE DIABATIC CASE}

This appendix outlines the derivation of Eq. (18) from Eq. (17). To this end, we expand each of the three $\epsilon$-dependent factors that appear in Eq. (17) in powers of $\epsilon$,

$$
\begin{aligned}
& \exp \left[-i \int_{t}^{t+\epsilon} d \tau U(\tau) / \hbar\right] \\
& \quad=1-\frac{i}{\hbar} \epsilon U(t)+\cdots, \\
& \left\langle s_{i}\left|e^{-i \epsilon \hat{H}_{s} / \hbar}\right| s_{k}\right\rangle\left\langle s_{l}\left|e^{i \epsilon \hat{H}_{s} / \hbar}\right| s_{j}\right\rangle \\
& =\delta_{i, k} \delta_{l, j}+\frac{i}{\hbar} \epsilon\left(\delta_{i, k}\left\langle s_{l}\left|\hat{H}_{s}\right| s_{j}\right\rangle-\delta_{l, j}\left\langle s_{i}\left|\hat{H}_{s}\right| s_{k}\right\rangle\right)+\cdots,
\end{aligned}
$$

$$
\begin{aligned}
\delta\left(Q-Q_{t+\epsilon}\right) \delta\left(P-P_{t+\epsilon}\right) & \\
= & \delta\left(Q-Q_{t}\right) \delta\left(P-P_{t}\right)-\epsilon\left\{\delta\left(P-P_{t}\right) \delta^{\prime}\left(Q-Q_{t}\right)\right. \\
& \left.\times \frac{\partial H_{\mathrm{av}}}{\partial P}-\delta\left(Q-Q_{t}\right) \delta^{\prime}\left(P-P_{t}\right) \frac{\partial H_{\mathrm{av}}}{\partial Q}\right\}+\cdots
\end{aligned}
$$

Equation (18) can be obtained by substituting those expansions back into Eq. (17), and calculating the overall firstorder term in $\epsilon$. The first-order term in Eq. (A1) gives rise to the term $-(i / \hbar)\left[V_{b s}\left(Q, s_{i}\right)-V_{b s}\left(Q, s_{j}\right)\right] \sigma_{i j}(Q, P ; t)$; the first-order term in Eq. (A2) gives rise to the term $-(i / \hbar) \Sigma_{k}\left[\left\langle s_{i}\left|\hat{H}_{s}\right| s_{k}\right\rangle \sigma_{k l}(Q, P ; t)-\sigma_{i k}(Q, P ; t)\left\langle s_{k}\left|\hat{H}_{s}\right| s_{j}\right\rangle\right]$; and the first-order term in Eq. (A3) gives rise to the term $\left\{H_{i j}^{\mathrm{av}}, \sigma_{i j}(Q, P ; t)\right\}$.

\section{APPENDIX B: THE DERIVATION OF THE MQCL EQUATION OF MOTION IN THE ADIABATIC CASE}

This appendix outlines the derivation of Eq. (36) from Eq. (29). To this end, we apply the linearization approximation to the relevant terms [see Eqs. (31)-(35)], and find the overall first-order term in the expansion of Eq. (29) in powers of $\epsilon$.
The $\epsilon$ dependence of the integrand in Eq. (29) is contained in a bath-dependent factor $\left\langle Q+\Delta / 2\left|e^{-i \epsilon \hat{H}_{b} / \hbar}\right| Q^{\prime}\right.$ $\left.+\Delta^{\prime} / 2\right\rangle\left\langle Q^{\prime}-\Delta^{\prime} / 2\left|e^{i \epsilon \hat{H}_{b} / \hbar}\right| Q-\Delta / 2\right\rangle$, and a systemdependent factor $\left\langle l\left(Q^{\prime}\right)\left|e^{-i \epsilon \hat{H}_{a}\left(Q^{\prime}+\Delta^{\prime} / 2\right) / \hbar}\right| k\left(Q^{\prime}\right)\right\rangle$ $\times\left\langle m\left(Q^{\prime}\right)\left|e^{i \epsilon \hat{H}_{a}\left(Q^{\prime}-\Delta^{\prime} / 2\right) / \hbar}\right| n\left(Q^{\prime}\right)\right\rangle$. The contribution to Eq. (36) that arises from the zero-order system term and firstorder bath term is given by

$$
\begin{aligned}
\frac{1}{2 \pi \hbar} & \sum_{k, m} \int d Q^{\prime} \int d P^{\prime} \int d \Delta \int d \Delta^{\prime} e^{-i P \Delta / \hbar} e^{i P^{\prime} \Delta^{\prime} / \hbar} \\
& \times\left\langle i(Q) \mid k\left(Q^{\prime}\right)\right\rangle\left\langle m\left(Q^{\prime}\right) \mid j(Q)\right\rangle \\
& \times\left\langle Q+\Delta / 2\left|e^{-i \epsilon \hat{H}_{b} / \hbar}\right| Q^{\prime}+\Delta^{\prime} / 2\right\rangle \\
& \times\left\langle Q^{\prime}-\Delta^{\prime} / 2\left|e^{i \epsilon \hat{H}_{b} / \hbar}\right| Q-\Delta / 2\right\rangle \sigma_{k m}^{W}\left(Q^{\prime}, P^{\prime} ; t\right) .
\end{aligned}
$$

We start by considering the term that corresponds to $k=i$ and $m=j$ in Eq. (B1),

$$
\begin{aligned}
\frac{1}{2 \pi \hbar} & \int d Q^{\prime} \int d P^{\prime} \int d \Delta \int d \Delta^{\prime} e^{-i P \Delta / \hbar} e^{i P^{\prime} \Delta^{\prime} / \hbar} \\
& \times\left\langle Q+\Delta / 2\left|e^{-i \epsilon \hat{H}_{b} / \hbar}\right| Q^{\prime}+\Delta^{\prime} / 2\right\rangle \\
& \times\left\langle Q^{\prime}-\Delta^{\prime} / 2\left|e^{i \epsilon \hat{H}_{b} / \hbar}\right| Q-\Delta / 2\right\rangle \sigma_{i j}^{W}\left(Q^{\prime}, P^{\prime} ; t\right) \\
\approx & \int d Q^{\prime} \int d P^{\prime} \sigma_{i j}^{W}\left(Q^{\prime}, P^{\prime} ; t\right) \delta\left(Q^{\prime}-Q-\epsilon P / M\right) \\
& \times \delta\left(P^{\prime}-P_{t}+\epsilon V_{b}^{\prime}\left(Q^{\prime}\right)\right),
\end{aligned}
$$

where the linearization approximation in Eq. (35) has been employed. Expanding the $\delta$ functions to first order in $\epsilon$ then yields the following contribution to Eq. (36):

$$
\left[-\frac{P}{M} \frac{\partial}{\partial Q}+\frac{\partial V_{b}}{\partial Q} \frac{\partial}{\partial P}\right] \sigma_{i j}^{W}(Q, P ; t) .
$$

Next, consider the term that corresponds to $k \neq i$ and $m$ $=j$ in Eq. (B1),

$$
\begin{aligned}
& \frac{1}{2 \pi \hbar} \sum_{k} \int d Q^{\prime} \int d P^{\prime} \int d \Delta \int d \Delta^{\prime} e^{-i P \Delta / \hbar} e^{i P^{\prime} \Delta^{\prime} / \hbar} \\
& \quad \times\left\langle i(Q) \mid k\left(Q^{\prime}\right)\right\rangle\left\langle Q+\Delta / 2\left|e^{-i \epsilon \hat{H}_{b} / \hbar}\right| Q^{\prime}+\Delta^{\prime} / 2\right\rangle \\
& \quad \times\left\langle Q^{\prime}-\Delta^{\prime} / 2\left|e^{i \epsilon \hat{H}_{b} / \hbar}\right| Q-\Delta / 2\right\rangle \sigma_{k j}^{W}(Q, P ; t),
\end{aligned}
$$

where we have used Eq. (31) in order to substitute $\left\langle j\left(Q^{\prime}\right) \mid j(Q)\right\rangle=1$. According to Eq. (32), the first nonvanishing term in the expansion of $\left\langle i(Q) \mid k\left(Q^{\prime}\right)\right\rangle$ is of first order in $\epsilon$. Thus, the first-order term in the expansion of Eq. (B4) in powers of $\epsilon$ involves the following zero-order bath term:

$$
\begin{aligned}
& {\left[\langle Q + \Delta / 2 | e ^ { - i \epsilon \hat { H } _ { b } / \hbar } | Q ^ { \prime } + \Delta ^ { \prime } / 2 \rangle \left\langleQ^{\prime}-\Delta^{\prime} / 2\left|e^{i \epsilon \hat{H}_{b} / \hbar}\right|\right.\right.} \\
& \quad \times Q-\Delta / 2\rangle]_{\epsilon=0}=\delta\left[Q-Q^{\prime}+\left(\Delta-\Delta^{\prime}\right) / 2\right] \\
& \quad \times \delta\left[Q-Q^{\prime}-\left(\Delta-\Delta^{\prime}\right) / 2\right]=\delta\left(Q-Q^{\prime}\right) \delta\left(\Delta-\Delta^{\prime}\right) .
\end{aligned}
$$

Thus, the corresponding contribution to Eq. (36) is given by

$$
-\sum_{k \neq i} d_{i k} \frac{P_{t}}{M} \sigma_{k j}^{W}(Q, P ; t) .
$$


Similarly, the term that corresponds to $k=i$ and $m \neq j$ in Eq. (B1) leads to the following contribution to Eq. (36):

$$
\sum_{k \neq j} d_{k j} \frac{P_{t}}{M} \sigma_{i k}^{W}(Q, P ; t) .
$$

It should be noted that the terms in Eq. (B1) that correspond to $k \neq i$ and $m \neq j$ do not contribute to Eq. (36), since the first nonvanishing term in the expansion is of second order in $\epsilon$.

The contribution to Eq. (36) that arises from the zeroorder bath term and first-order system term can be put in the following form:

$$
\begin{aligned}
\frac{1}{2 \pi \hbar} & \sum_{k, m} \int d P^{\prime} \int d \Delta e^{-i\left(P-P^{\prime}\right) \Delta / \hbar} \\
& \times\left\langle i(Q)\left|e^{-i \epsilon \hat{H}_{a}(Q+\Delta / 2) / \hbar}\right| k(Q)\right\rangle \\
& \times\left\langle m(Q)\left|e^{i \epsilon \hat{H}_{a}(Q-\Delta / 2) / \hbar}\right| j(Q)\right\rangle \sigma_{k m}^{W}\left(Q, P^{\prime} ; t\right) .
\end{aligned}
$$

The term in the sum that correspond to $k=i$ and $m=j$ then makes the following contribution to Eq. (36):

$$
\begin{gathered}
-i \omega_{i j}(Q) \sigma_{i j}^{W}(Q, P ; t)+\frac{1}{2}\left(\left\langle i(Q)\left|\frac{V_{b s}}{\partial Q}\right| i(Q)\right\rangle\right. \\
\left.+\left\langle j(Q)\left|\frac{V_{b s}}{\partial Q}\right| j(Q)\right\rangle\right) \frac{\partial \sigma_{i j}^{W}(Q, P ; t)}{\partial P} .
\end{gathered}
$$

The terms in the sum that correspond to $k \neq i$ and $m=j$ or $k=i$ and $m \neq j$ make the following contributions to Eq. (36):

$$
\begin{aligned}
& \sum_{k \neq i} \frac{1}{2}\left\langle k(Q)\left|\frac{V_{b s}}{\partial Q}\right| j(Q)\right\rangle \frac{\partial \sigma_{k j}^{W}(Q, P ; t)}{\partial P}, \\
& \sum_{k \neq j} \frac{1}{2}\left\langle i(Q)\left|\frac{V_{b s}}{\partial Q}\right| m(Q)\right\rangle \frac{\partial \sigma_{i m}^{W}(Q, P ; t)}{\partial P} .
\end{aligned}
$$

Finally, as was noted above, terms with $k \neq i$ and $m \neq j$ do not contribute to Eq. (36).

${ }^{1}$ B. J. Berne, G. Ciccotti, and D. F. Coker, Eds. Classical and Quantum Dynamics in Condensed Phase Simulations (World Scientific, London, 1998).

${ }^{2}$ N. Makri, Annu. Rev. Phys. Chem. 50, 167 (1999).

${ }^{3}$ P. Jungwirth and R. B. Gerber, Chem. Rev. (Washington, D.C.) 99, 1583 (1999).

${ }^{4}$ R. P. Feynman and A. R. Hibbs, Quantum Mechanics and Path Integrals (McGraw-Hill, New York, 1965).

${ }^{5}$ L. S. Schulman, Techniques and Applications of Path Integration (Wiley, New York, 1981).

${ }^{6}$ H. Kleinert, Path Integrals in Quantum Mechanics, Statistics and Polymer Physics (World Scientific, New Jersey, 1995).

${ }^{7}$ R. P. Feynman and F. L. Vernon, Jr., Ann. Phys. (Leipzig) 24, 118 (1963).

${ }^{8}$ P. G. Wolynes, Phys. Rev. Lett. 47, 968 (1981).

${ }^{9}$ A. O. Caldeira and A. J. Leggett, Ann. Phys. (Leipzig) 149, 374 (1983).

${ }^{10}$ R. D. Coalson, J. Chem. Phys. 86, 995 (1987).

${ }^{11}$ C. Mak and D. Chandler, Phys. Rev. A 44, 2352 (1991).

${ }^{12}$ M. Topaler and N. Makri, J. Chem. Phys. 101, 7500 (1994).

${ }^{13}$ M. Topaler and N. Makri, J. Chem. Phys. 97, 9001 (1992).

${ }^{14}$ M. Topaler and N. Makri, Chem. Phys. Lett. 210, 285 (1993).

${ }^{15}$ M. Topaler and N. Makri, Chem. Phys. Lett. 210, 448 (1993).

${ }^{16}$ M. Topaler and N. Makri, J. Phys. Chem. 100, 4430 (1996).

${ }^{17}$ D. E. Makarov and N. Makri, Chem. Phys. Lett. 221, 482 (1994).

${ }^{18}$ N. Makri and D. Makarov, J. Chem. Phys. 102, 4600 (1995).

${ }^{19}$ N. Makri and D. Makarov, J. Chem. Phys. 102, 4611 (1995).
${ }^{20}$ N. Makri, J. Math. Phys. 36, 2430 (1995).

${ }^{21}$ N. Makri, E. Sim, D. E. Makarov, and M. Topaler, Proc. Natl. Acad. Sci. U.S.A. 93, 3926 (1996).

${ }^{22}$ E. Sim and N. Makri, Comput. Phys. Commun. 99, 335 (1997).

${ }^{23}$ E. Sim and N. Makri, J. Phys. Chem. B 101, 5446 (1997).

${ }^{24}$ N. Makri, J. Phys. Chem. A 102, 4414 (1998).

${ }^{25}$ J. S. Shao and N. Makri, J. Chem. Phys. 116, 507 (2002).

${ }^{26}$ A. A. Golosov, R. A. Friesner, and P. Pechukas, J. Chem. Phys. 110, 138 (1999).

${ }^{27}$ A. A. Golosov, R. A. Friesner, and P. Pechukas, J. Chem. Phys. 112, 2095 (2000).

${ }^{28}$ N. Makri and K. Thompson, Chem. Phys. Lett. 291, 101 (1998).

${ }^{29}$ K. Thompson and N. Makri, J. Chem. Phys. 110, 1343 (1999).

${ }^{30}$ K. Thompson and N. Makri, Phys. Rev. E 59, R4729 (1999).

${ }^{31}$ S. Nakajima, Prog. Theor. Phys. 20, 948 (1958).

${ }^{32}$ R. Zwanzig, Lect. Theor. Phys. 3, 106 (1960).

${ }^{33}$ R. Zwanzig, J. Chem. Phys. 33, 1338 (1960).

${ }^{34}$ R. Zwanzig, Physica (Amsterdam) 30, 1109 (1984).

${ }^{35}$ I. Prigogine and P. Resibois, Physica (Amsterdam) 27, 629 (1961).

${ }^{36}$ F. Haake, Springer Tracts Mod. Phys. 66, 98 (1973).

${ }^{37}$ H. Grabert, Projection Operator Techniques in Nonequilibrium Statistical Mechanics (Springer, Berlin, 1982).

${ }^{38}$ R. Alicki and K. Lendi, Quantum Dynamical Semigroups and Applications (Springer, Berlin, 1987).

${ }^{39}$ V. May and O. Kühn, Charge and Energy Transfer Dynamics in Molecular Systems (Wiley-VCH, Berlin, 2000).

${ }^{40}$ B. Yoon, J. M. Deutch, and J. H. Freed, J. Chem. Phys. 62, 4687 (1975).

${ }^{41}$ I. Oppenheim, K. E. Shuler, and G. H. Weiss, Stochastic Processes in Chemical Physics: The Master Equation (MIT, Cambridge, MA, 1977).

${ }^{42}$ S. Mukamel, I. Oppenheim, and J. Ross, Phys. Rev. A 17, 1988 (1978).

${ }^{43}$ V. Romero-Rochin and I. Oppenheim, Physica A 155, 52 (1989).

${ }^{44}$ V. Romero-Rochin, A. Orsky, and I. Oppenheim, Physica A 156, 244 (1989).

${ }^{45}$ Q. Shi and E. Geva, J. Chem. Phys. 119, 12045 (2003).

${ }^{46}$ M. P. Miller and C. W. McCurdy, J. Chem. Phys. 69, 5163 (1978).

${ }^{47}$ C. W. Mccurdy, H. D. Meyer, and W. H. Miller, J. Phys. Chem. 70, 3214 (1978).

${ }^{48}$ H. D. Meyer and W. H. Miller, J. Chem. Phys. 70, 3214 (1979).

${ }^{49}$ H. D. Meyer and W. H. Miller, J. Chem. Phys. 71, 2156 (1979).

${ }^{50}$ G. Stock and M. Thoss, Phys. Rev. Lett. 78, 578 (1997).

${ }^{51}$ X. Sun, H. Wang, and W. H. Miller, J. Chem. Phys. 109, 4190 (1998).

${ }^{52}$ H. Wang, X. Song, D. Chandler, and W. H. Miller, J. Chem. Phys. 110, 4828 (1999).

${ }^{53}$ W. H. Miller, J. Phys. Chem. A 105, 2942 (2001).

${ }^{54}$ A. Schmid, J. Low Temp. Phys. 49, 609 (1982).

${ }^{55}$ R. E. Cline, Jr. and P. G. Wolynes, J. Chem. Phys. 88, 4334 (1988).

${ }^{56}$ H. Kleinert and S. V. Shabanov, Phys. Lett. A 200, 224 (1995).

${ }^{57}$ Q. Shi and E. Geva, J. Chem. Phys. 120, 10647 (2004).

${ }^{58}$ H. Wang, X. Sun, and W. H. Miller, J. Chem. Phys. 108, 9726 (1998).

${ }^{59}$ Q. Shi and E. Geva, J. Phys. Chem. A 107, 9059 (2003).

${ }^{60}$ Q. Shi and E. Geva, J. Phys. Chem. A 107, 9070 (2003).

${ }^{61}$ E. Rabani, G. Krilov, and B. J. Berne, J. Chem. Phys. 112, 2605 (2000).

${ }^{62}$ Q. Shi and E. Geva, J. Phys. Chem. A (in press).

${ }^{63}$ J. C. Tully, Faraday Discuss. 110, 407 (1998).

${ }^{64}$ R. B. Gerber, V. Buch, and M. A. Ratner, J. Chem. Phys. 77, 3022 (1982).

${ }^{65}$ G. D. Billing, J. Chem. Phys. 99, 5849 (1993).

${ }^{66}$ J. C. Tully and R. K. Preston, J. Chem. Phys. 55, 562 (1971).

${ }^{67}$ W. H. Miller and T. F. George, J. Chem. Phys. 56, 5637 (1972).

${ }^{68}$ J. C. Tully, J. Chem. Phys. 93, 1061 (1990).

${ }^{69}$ M. F. Herman, J. Chem. Phys. 103, 8081 (1995).

${ }^{70}$ D. F. Coker and L. Xiao, J. Chem. Phys. 102, 496 (1995).

${ }^{71}$ P. J. Kuntz, J. Chem. Phys. 95, 141 (1991).

${ }^{72}$ O. V. Prezhdo and P. J. Rossky, J. Chem. Phys. 107, 825 (1997).

${ }^{73}$ Y. L. Volobuev, M. D. Hack, M. S. Topaler, and D. G. Truhlar, J. Chem. Phys. 112, 9716 (2000).

${ }^{74}$ C. C. Martens and J. Fang, J. Chem. Phys. 106, 4918 (1997).

${ }^{75}$ A. Donoso and C. C. Martens, J. Phys. Chem. A 102, 4291 (1998).

${ }^{76}$ A. Donoso and C. C. Martens, J. Chem. Phys. 112, 3980 (2000).

${ }^{77}$ A. Donoso, D. Kohen, and C. C. Martens, J. Chem. Phys. 112, 7345 (2000).

${ }^{78}$ C. Schütte, Konard-Zuse-Zentrum für informationstechnik Belin Preprint SC 99 (June 1999).

${ }^{79}$ M. Santer, U. Manthe, and G. Stock, J. Chem. Phys. 114, 2001 (2001).

${ }^{80}$ R. Kapral and G. Ciccotti, J. Chem. Phys. 110, 8919 (1999). 
${ }^{81}$ S. Nielsen, R. Kapral, and G. Ciccotti, J. Chem. Phys. 112, 6543 (2000).

${ }^{82}$ C. Wan and J. Schofield, J. Chem. Phys. 113, 7047 (2000).

${ }^{83}$ C. Wan and J. Schofield, J. Chem. Phys. 112, 4447 (2000).

${ }^{84}$ A. Donoso and C. C. Martens, J. Chem. Phys. 116, 10598 (2002).
${ }^{85}$ K. Thompson and N. Makri, Chem. Phys. Lett. 291, 101 (1998).

${ }^{86}$ Q. Shi and E. Geva, J. Chem. Phys. 118, 8173 (2003).

${ }^{87}$ S. Mukamel, Principles of Nonlinear Optical Spectroscopy (Oxford, New York, 1995). 